\title{
Experimentally induced innovations lead to persistent culture via conformity in wild birds
}

\author{
Lucy M. Aplin ${ }^{1,2}$, Damien R. Farine ${ }^{1,3,4}$, Julie Morand-Ferron ${ }^{5}$, Andrew Cockburn ${ }^{2}$, Alex Thornton ${ }^{6}$ \& Ben C. Sheldon ${ }^{1,7}$
}

In human societies, cultural norms arise when behaviours are transmitted through social networks via high-fidelity social learning ${ }^{1}$. However, a paucity of experimental studies has meant that there is no comparable understanding of the process by which socially transmitted behaviours might spread and persist in animal populations ${ }^{2,3}$. Here we show experimental evidence of the establishment of foraging traditions in a wild bird population. We introduced alternative novel foraging techniques into replicated wild sub-populations of great tits (Parus major) and used automated tracking to map the diffusion, establishment and long-term persistence of the seeded innovations. Furthermore, we used social network analysis to examine the social factors that influenced diffusion dynamics. From only two trained birds in each sub-population, the information spread rapidly through social network ties, to reach an average of $75 \%$ of individuals, with a total of 414 knowledgeable individuals performing 57,909 solutions over all replicates. The sub-populations were heavily biased towards using the technique that was originally introduced, resulting in established local traditions that were stable over two generations, despite a high population turnover. Finally, we demonstrate a strong effect of social conformity, with individuals disproportionately adopting the most frequent local variant when first acquiring an innovation, and continuing to favour social information over personal information. Cultural conformity is thought to be a key factor in the evolution of complex culture in humans ${ }^{4-7}$. In providing the first experimental demonstration of conformity in a wild non-primate, and of cultural norms in foraging techniques in any wild animal, our results suggest a much broader taxonomic occurrence of such an apparently complex cultural behaviour.

Social learning, in which animals learn from others, can enable novel behaviours to spread between individuals, creating group-level behaviours, including traditions and culture ${ }^{6,8,9}$. Social transmission occurs between interacting individuals; hence, group dynamics and population structure will determine the spread and persistence of traditions $s^{2,3,9-11}$ Additionally, individuals may strategically use social learning to maximize its adaptive value, with consequences for when, how and what traditions are established ${ }^{4,12}$. However, while the capacity for social learning has been described in many phylogenetically diverse taxa ${ }^{13}$ and has been detailed in comprehensive laboratory studies ${ }^{13-15}$, we have little knowledge of the social dynamics associated with such learning in natural systems. Experimentally quantifying cultural transmission in wild populations remains difficult, with limitations associated with isolating and training individuals ${ }^{5}$, tracking the spread of information across large numbers of animals ${ }^{14}$ and eliminating alternative explanations such as individual trial-and-error learning ${ }^{8,14}$.

Early observational studies of tits provide one of the most widely cited examples of animal innovation and culture, when British birds famously began to pierce the foil caps of milk bottles to take the cream ${ }^{16-18}$. More generally, great tits (P. major) are known to be highly innovative, opportunistic foragers ${ }^{19}$ and to use social information in a wide range of contexts ${ }^{20}$.
This life history, coupled with their fission-fusion social structure ${ }^{21}$, makes them excellent models for a large-scale empirical investigation of the social processes associated with cultural transmission. Here we used a novel system that incorporates automated data collection and passive integrated transponder tags, together with recently developed methods for social network analysis, to investigate the spread, establishment and persistence of experimentally seeded traditions in wild great tits.

We first developed an automated puzzle box that is baited with live mealworms (Fig. 1a), and performed a cultural diffusion experiment based on the two-action and control design ${ }^{14}$ but where treatment groups were exposed to a demonstrator trained on one of two distinct but equivalent actions. Two resident males were caught from each of eight subpopulations and exposed to one of three training regimens in captivity. In the first condition ('control'), for which there were three sub-populations (that is, three replicates), neither individual was given any training. In the second condition ('option A'; two replicates), both individuals were trained to access food from the puzzle box by using their bill to move the blue side of the sliding door from left to right. Last, in the third condition ('option B'; three replicates), the birds were trained to solve the puzzle box by moving the red side of the sliding door from right to left (Supplementary Video 1). After 4 days of training, all birds were released back into the wild, and three puzzle boxes, with both options available, were installed $250 \mathrm{~m}$ apart in each sub-population (Extended Data Fig. 1). We then automatically monitored the individual visits to, and solutions ('solves') at these puzzle boxes, over short-term (20 days of exposure over 4 weeks) and long-term ( 5 days of exposure, 9 months later) periods.

In the five sub-populations that were seeded with trained demonstrators, knowledge of how to solve the novel puzzle spread rapidly over 20 days of exposure (Fig. 1b). A mean of $75 \%$ of the members of each local population (68-83\%, $n=37-96)$ solved the puzzle box at least once. The diffusion of this behaviour was clearly sigmoidal (sigmoidal versus linear fit, change in Akaike information criterion $(\Delta \mathrm{AIC})=15.31-54.17)$, except in one replicate $(\mathrm{T} 5, \Delta \mathrm{AIC}=0.13)$. By contrast, many fewer individuals solved the puzzle box in control sub-populations $(9-53 \%$, $n=5-46$; Fig. 1b), in which uptake initially relied on individual innovation. The latency to the first solve, excluding the demonstrator, was significantly longer in control areas than in treatment areas (Welch's two-sample $t$-test, $t_{(6)}=-16.1, P<0.01$; Fig. $1 \mathrm{~b}$ ), and the total number of solutions was significantly lower $\left(t_{(6)}=4.6, P=0.02\right.$; Fig. 1c). There was a striking difference between the replicates that were seeded with alternative solving techniques. In all treatment sub-populations, learning was heavily biased towards the technique that was originally demonstrated $\left(t_{(8)}=9.7, P<0.01\right.$; Fig. $\left.1 \mathrm{c}\right)$, while no consistent side bias was observed between the control sub-populations $\left(_{(4)}=-0.03\right.$, $P=0.97$; Fig. 1c).

We derived the social network for each sub-population independently of the social learning experiment, with 10 days' sampling at a grid of

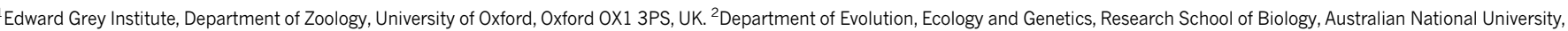

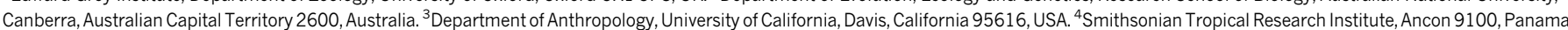

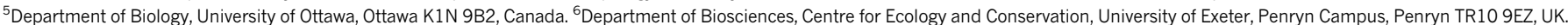

${ }^{7}$ Department of Ecology and Genetics, Uppsala University, 75236 Uppsala, Sweden. 


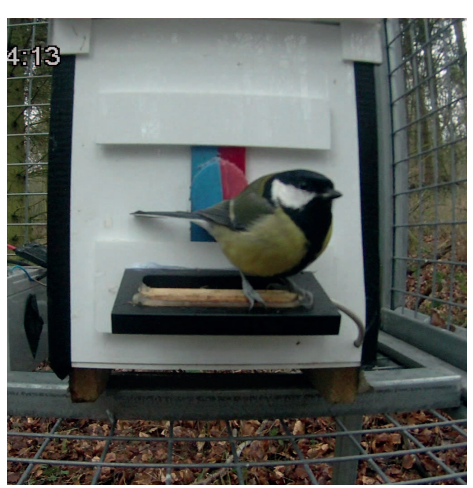

b

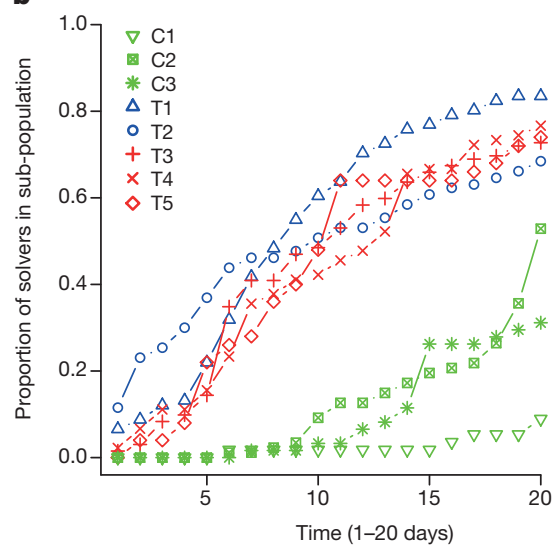

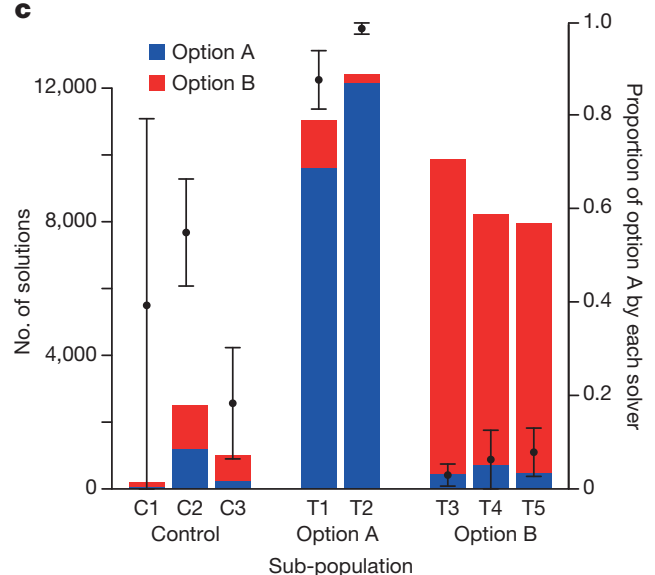

Figure $1 \mid$ Cultural diffusion experiment. a, A puzzle box in which birds can slide the door open in two directions (from the left, option A; or the right, option B) to access a reward. The puzzle box records the identity, visit duration and solution choice, and it resets after each visit. $\mathbf{b}$, Diffusion curves for the treatment sub-populations, with demonstrators (T1-5; $n=91,130,132,50,90$, respectively), and the control sub-populations, without demonstrators (C1-3;

sunflower-seed feeders that had been equipped to record visitation data (Extended Data Fig. 2a, b). Co-occurrences (see Methods) were detected using a Gaussian mixture model to isolate clusters of visits in the spatiotemporal data streams ${ }^{22}$, with repeated foraging associations between individuals forming the basis of social networks (Extended Data Fig. 2b, c). The social networks for all replicates were significantly non-random, even at the most local scale (T1-5, $P<0.001)$, and network-based diffusion analysis was used to quantify the extent to which these social ties predicted the acquisition of behaviour ${ }^{23}$. From pooled replicate data, a network diffusion model that included social transmission was overwhelmingly supported over asocial learning $(\triangle \mathrm{AIC}=1520.7)$; the learning rate was estimated to increase by a factor of 12.0 per unit of association with knowledgeable individuals (Extended Data Fig. 3). An effect of age and sex was also supported, with juveniles and males having a faster learning rate (Table 1). These results support a dominant effect of social learning on the emergence of this novel behaviour and also demonstrate that the diffusion of innovation was influenced by the fine-scale patterns of social interactions (Supplementary Video 3).

In all of the experimental replicates, the alternative solution, which was equally difficult and equally rewarded, was performed by at least one individual within the first 6 days of exposure (median, day 4). However, in contrast to most previous studies, in which discovery of an alternative solution led to the progressive erosion of the use of the seeded variant behaviour ${ }^{2,5,24}$, we observed a pronounced strengthening of traditions $n=56,87,61$, respectively).c, The total number of solutions using each option in each replicate (sub-population) (left $y$ axis, shown as stacked bars). The average proportion (dots) of option A performed by individuals, with 95\% CI (bars) is shown (right $y$ axis). The total number of solvers was 5, 46 and 19 for C1, C2 and C3 (controls), respectively; 76 and 89 for T1 and T2 (option A), respectively; and 96, 37 and 69 for T3, T4 and T5 (option B), respectively.

over the rest of the experiment. To analyse this change in behaviour over time, we used a generalized estimating equation model $^{2}$ where the dependent variable was the proportion of solutions using the seeded technique on each day of data collection and the explanatory variables were individuals and replicates. From pooled replicate data, there was strong evidence that the preference for the arbitrary tradition increased over time (coefficient \pm s.e.m. $=0.13 \pm 0.02, P<0.001$ ), with an estimated $14 \%$ increase in bias per day $(95 \%$ confidence interval $(\mathrm{CI})=$ $8-18 \%$; Fig. 2a). This finding is consistent with a conformist transmission bias, with individuals preferentially adopting the more commonly practised variant when solving the puzzle box ${ }^{5,7,25,26}$. More conclusive evidence for such positive frequency-dependent copying ${ }^{25}$ was observed when only the first solution for each individual was considered, with birds disproportionately likely to initially adopt the variant used by the majority of their group (sigmoidal versus linear fit, $\Delta \mathrm{AIC}=38.34$; Fig. 2b).

Individuals thus preferentially learnt the most common option when first learning (Fig. 2b). Yet, remarkably, they also continued to prioritize social information over personal information, matching their behaviour to the common variant even after experiencing an equally rewarding alternative. We analysed the trajectories for those individuals that used both options $(n=78)$. The majority of these individuals $(85 \%$, $n=66$ ) retained a preference for the seeded variant (for example, see Fig. 2c and Extended Data Fig. 4). Three birds had a strong preference for the uncommon variant, and eight birds switched from the alternative

Table 1 | Network-based diffusion analysis

\begin{tabular}{lllll}
\hline Network-based diffusion model outputs & & & & \\
\hline Transmission model & $\Delta$ AIC (top model) & $\Sigma \omega_{i}$ & Social transmission parameter (estimated) & $95 \% \mathrm{Cl}$ \\
\hline Social: multiplicative & 0 & 0.99 & 12.0 & $8.8-16.0$ \\
T1 & - & - & 22.4 & $11.8-30.2$ \\
T2 & - & - & 12.2 & $8.2-17.1$ \\
T3 & - & - & 7.3 & $2.9-14.3$ \\
T4 & - & - & 29.8 & $10.9-42.6$ \\
T5 & - & - & 13.4 & $8.3-20.02$ \\
Social: additive & 33.7 & 0.01 & - & - \\
Asocial & 1520.7 & & (Constrained to 0) & - \\
\hline Individual-level effects & & & \\
\hline Variable & $\Delta$ AlC (top model) & $\Sigma \omega_{i}$ & Estimate & \\
\hline Age (juvenile or adult) & 0 & 0.99 & -0.18 & 0.70 \\
Sex (F or M) & 0 & 0.97 & 0.10 & 1.22 \\
Natal origin (resident or immigrant) & 3.9 & 0.13 & 0.07 & 1.16 \\
\hline
\end{tabular}

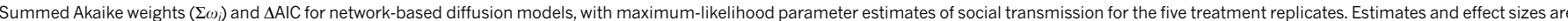

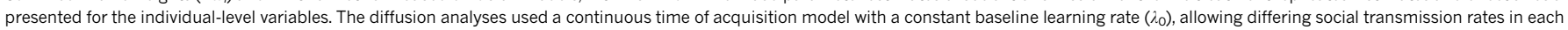
replicate. F, female; immigrant, dispersed into the study site; M, male; resident, locally born. 

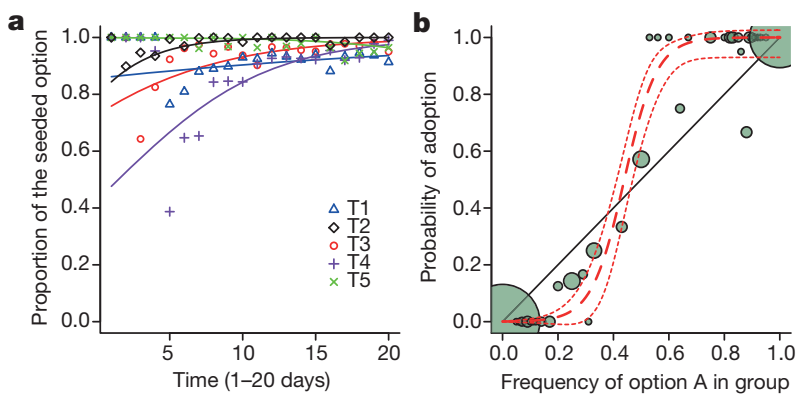

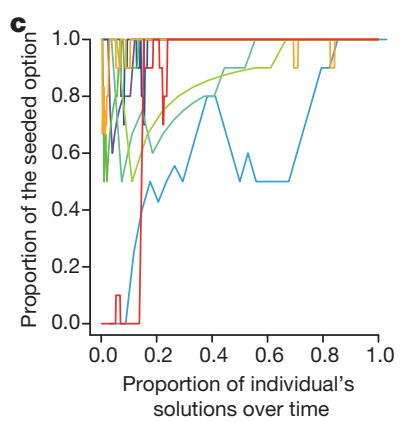

Figure $2 \mid$ Evidence of social conformity. a, The proportion of solutions using the seeded technique increased significantly over time in each replicate. The points are the proportion of solutions using the seeded technique on each day; the lines show the generalized estimating equation model fit. $\mathbf{b}$, Comparison of the frequency of option A in the sub-population with an individual's first learnt option (pooled replicate data from T1-5). The node size represents the

variant to the common variant. However, none of the birds made the reciprocal switch, and only one individual had no significant preference. A subset of birds that dispersed between the experimental replicates (a total of $n=41$, of which 24 were between the two years of the experiment, see below) provided additional evidence. Of 27 birds that moved between replicates with the same seeded tradition, 26 (96\%) retained their preference for the common variant. In contrast, of 14 individuals that moved between replicates with different seeded traditions, $10(71 \%)$ changed their behaviour to match the common variant in the new location, 3 retained their initial preference and 1 showed no preference $\left(\chi_{(1)}^{2}=21.6, P<0.001\right)$.

Seeded arbitrary traditions thus formed and persisted in each subpopulation (Fig. 2). To investigate the long-term stability of these traditions, we re-installed the puzzle boxes in one replicate of each condition (T1, T3 and C1) over 5 days in the following winter. Substantial turnover in the population had occurred owing to the high mortality rates typical of this species ${ }^{27}$; on average, only $40 \%$ of each sub-population had been present the previous year. No additional demonstrators were trained, and no individual had had contact with the puzzle box in the intervening months. In the control sub-population, all solves $(n=42)$ were performed by only three individuals, all of which had also solved the puzzle box the previous year. However, in the two experimental subpopulations, knowledge of how to solve the puzzle box emerged even number of individuals ( $n=1-147)$. The black line shows the expected result under unbiased copying; the central red line shows the model fit with $95 \%$ CI (outer red lines). c, The solution trajectories for individuals in the T2 sub-population that used both possible options $(n=10)$. The lines are the running average of the proportion of option $\mathrm{A}$ for each individual over the last ten visits, with each colour representing a single individual.

faster than it had the preceding year, both among prior solvers and birds that were inexperienced at the task: in T1, 29 individuals solved the puzzle box a total of 967 times, and in T3, 35 individuals solved the puzzle box a total of 2,329 times (Fig. 3b). The results suggest a strong initial effect of memory, followed by a rapid, oblique transmission facilitated by the greater number of demonstrators than in the initial experiment: on the first day of exposure, $60 \%$ (T1) and $82 \%$ (T3) of 'solvers' were birds that had solved the puzzle box in the initial experiment, outweighing their representation in the general population (36\% in $\mathrm{T} 1$ and $46 \%$ in T3). The sub-populations also retained their original technique, with the solutions being heavily biased towards the option that had been seeded in the original experiment (Fig. 3b). Intriguingly, among birds that were present in both years, the within-individual bias towards the seeded variant had increased (linear mixed model, $t_{(83)}=2.80, P<0.01$; Fig. $3 \mathrm{c}$ ), resulting in arbitrary traditions that were retained and strengthened.

In summary, we show that wild great tits use social learning to acquire novel behaviours and that foraging techniques introduced by few individuals (here only two in each replicate) can spread rapidly to the majority of the population, forming stable arbitrary traditions. Both social network ties and individual characteristics determined the transmission of these foraging techniques ${ }^{23}$. The introduced arbitrary traditions were stable over both short-term and long-term periods, becoming increasingly entrenched over two generations. This stability appeared to be a result

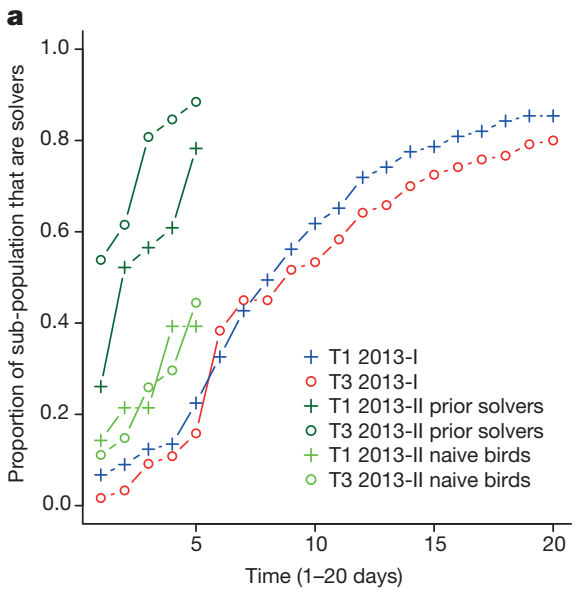

b

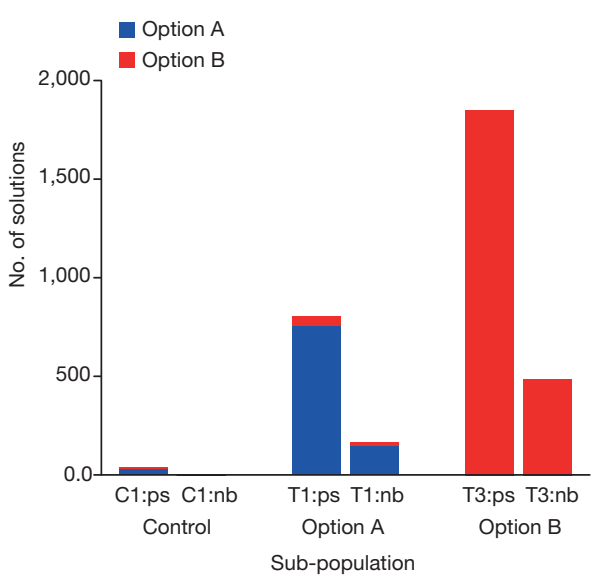

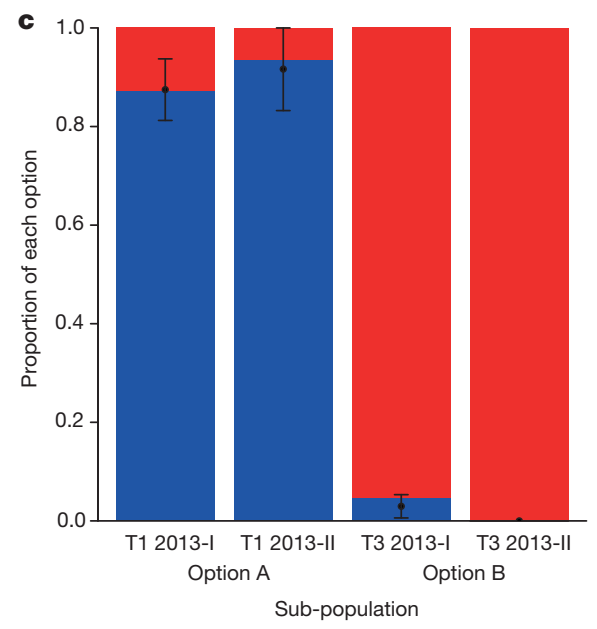

Figure 3 Local traditions persist across years. a, Diffusion curves for the initial exposure (T1 2013-I and T3 2013-I, where I is 20 days) and the second exposure (T1 2013-II and T3 2013-II, where II is 5 days, 9 months later).

The cumulative uptake of the behaviour in the second exposure is much higher for prior solvers (T1 2013-II and T3 2013-II, $n=23$ and 26, respectively) but is also higher for naive birds (T1 2013-II and T3 2013-II, $n=28$ and 27, respectively). b. The number of solutions using option A or B. In T1, one circuit board failed, so the data are derived from two of three devices. nb, native birds; ps, prior solvers. c, The proportion of option A or B used in the initial and second exposure. The histograms shows the data for the sub-populations; the dots show the mean proportion of option A performed by individuals, and error bars show the $95 \%$ CI. 
of informational conformity, with individuals matching their behaviour to the most common variant when first learning and then continuously updating their personal information. Conformity has long been considered a central component of human culture $\mathrm{e}^{25,26,28}$, but experimental evidence for its occurrence in wild animals has been limited to a study of food preferences in vervet monkeys ${ }^{5}$. We provide the first experimental demonstration, to our knowledge, of conformist transmission and cultural norms in foraging techniques in a wild animal. Our study argues against the previous view that such behaviour is restricted to the primate lineage $\mathrm{e}^{26,28-30}$ and calls for a reconsideration of the evolution and ecology of cultural conformity.

Online Content Methods, along with any additional Extended Data display items and Source Data, are available in the online version of the paper; references unique to these sections appear only in the online paper.

\section{Received 23 June; accepted 23 October 2014.}

Published online 3 December 2014.

1. Rogers, E. M. Diffusion of Innovations 4th edn (Free Press, 1995).

2. Claidière, N., Messer, E. J. E., Hoppitt, W. \& Whiten, A. Diffusion dynamics of socially learned foraging techniques in squirrel monkeys. Curr. Biol. 23, 1251-1255 (2013).

3. Cantor, M. \& Whitehead, H. The interplay between social networks and culture: theoretically and among whales and dol phins. Phil. Trans. R. Soc. B 368, 20120340 (2013).

4. Rendell, L. et al. Cognitive culture: theoretical and empirical insights into social learning strategies. Trends Cogn. Sci. 15, 68-76 (2011).

5. van de Waal, E, Borgeaud, C. \& Whiten, A. Potent social learning and conformity shape a wild primate's foraging decisions. Science 340, 483-485 (2013).

6. Whiten, A., Hinde, R. A., Laland, K. N. \& Stringer, C. B. Culture evolves. Phil. Trans. R. Soc. B 366, 938-948 (2011)

7. Whiten, A., Horner, V. \& de Waal, F. B. Conformity to cultural norms of tool use in chimpanzees. Nature 437, 737-740 (2005).

8. Laland, K. N. \& Janik, V. M. The animal cultures debate. Trends Ecol. Evol. 21, 542-547 (2006).

9. Warner, R. R. Traditionality of mating-site preferences in a coral reef fish. Nature 335, 719-721 (1988)

10. Coussi-Korbel, S. \& Fragaszy, D. M. On the relation between social dynamics and social learning. Anim. Behav. 50, 1441-1453 (1995).

11. Dean, L. G., Kendal, R. L., Schapiro, S. J., Thierry, B. \& Laland, K. N. Identification of the social and cognitive processes underlying human cumulative culture. Science 335, 1114-1118 (2012).

12. Laland, K. Social learning strategies. Learn. Behav. 32, 4-14 (2004).

13. Galef, B. G. \& Laland, K. N. Social learning in animals: empirical studies and theoretical models. Bioscience 55, 489-499 (2005).

14. Whiten, A. \& Mesoudi, A. Establishing an experimental science of culture: animal social diffusion experiments. Phil. Trans. R. Soc. B 363, 3477-3488 (2008).

15. Galef, B. G. in Oxford Handbook of Comparative Cognition (eds Zentall, T. R. \& Wasserman, E.) Ch. 40, 803-818 (Oxford Univ. Press, 2012).
16. Fisher, J. B. \& Hinde, R. A. The opening of milk bottles by birds. Br. Birds $\mathbf{4 2}$, 347-357 (1949).

17. Sherry, D. F. \& Galef, B. G. Cultural transmission without imitation: milk bottle opening by birds. Anim. Behav. 32, 937-938 (1984).

18. Aplin, L. M., Sheldon, B. \& Morand-Ferron, J. Milk-bottles revisited: social learning and individual variation in the blue tit (Cyanistes caeruleus). Anim. Behav. 85, 1225-1232 (2013)

19. Morand-Ferron, J., Cole, E. F., Rawles, J. E. C. \& Quinn, J. L. Who are the innovators? A field experiment with 2 passerine species. Behav. Ecol. 22, 1241-1248 (2011)

20. Slagsvold, T.\& Wiebe, K. L. Social learning in birds and its role in shaping a foraging niche. Phil. Trans. R. Soc. B 366, 969-977 (2011).

21. Aplin, L. M. et al. Individual personalities predict social behaviour in wild networks of great tits (Parus major). Ecol. Lett. 16, 1365-1372 (2013).

22. Psorakis, I., Roberts, S. J., Rezek, I. \& Sheldon, B. C. Inferring social network structure in ecological systems from spatio-temporal data streams. J. R. Soc. Interface 9, 3055-3066 (2012).

23. Allen, J., Weinrich, M. T., Hoppitt, W. \& Rendell, L. Network-based diffusion analysis reveals cultural transmission of lobtail feeding in humpback whales. Science $\mathbf{3 4 0}$ 485-488 (2013).

24. Thornton, A. \& Malapert, A. The rise and fall of an arbitrary tradition: an experiment with wild meerkats. Proc. R. Soc. B 276, 1269-1276 (2009).

25. Morgan, T. J. H. \& Laland, K. The biological bases of conformity. Front. Neurosci. 6, 87 (2012).

26. van Leeuwen, E. J. C. \& Haun, D. B. M. Conformity in nonhuman primates: fad or fact? Evol. Hum. Behav. 34, 1-7 (2013).

27. Bouwhuis, S., Choquet, R., Sheldon, B. C. \& Verhulst, S. The forms and fitness cost of senescence: age-specific recapture, survival, reproduction, and reproductive value in a wild bird population. Am. Nat. 179, E15-E27 (2012).

28. Haun, D. B. M., Rekers, Y. \& Tomasello, M. Majority-biased transmission in chimpanzees and human children, but not orangutans. Curr. Biol. 22, 727-731 (2012).

29. de Waal, F. B. Animal conformists. Science 340, 437-438 (2013).

30. van Schaik, C. P. Animal culture: chimpanzee conformity? Curr. Biol. 22, R402-R404 (2012)

Supplementary Information is available in the online version of the paper.

Acknowledgements This project was supported by grants from the BBSRC (BB/ L006081/1) and the ERC (AdG 250164) to B.C.S., who was also supported by a visiting professorship at Uppsala University. L.M.A. was also supported by an Australian Postgraduate Award; and A.T., by a BBSRC David Phillips Fellowship (BB/H021817/1). The EGl social networks group, S. Lang and K. McMahon provided assistance in the field, and M. Whitaker produced electronic components for the puzzle boxes.

Author Contributions The study was initially conceived by L.M.A., J.M.-F. and B.C.S., with input from D.R.F., A.C. and A.T. in designing the experiments. Infrastructure to support the work was conceived and developed by B.C.S. The experimental work was led by L.M.A.; and the analysis, by L.M.A. and D.R.F. The manuscript was drafted by L.M.A. and B.C.S., and important contributions were made by all of the other authors.

Author Information Reprints and permissions information is available at www.nature.com/reprints. The authors declare no competing financial interests. Readers are welcome to comment on the online version of the paper. Correspondence and requests for materials should be addressed to L.M.A. (lucy.aplin@zoo.ox.ac.uk). 


\section{METHODS}

Study population and area. The study was conducted in a wintering population of tits in Wytham Woods, UK (51 $46^{\prime} \mathrm{N}, 01^{\circ} 20^{\prime} \mathrm{W}$; Extended Data Fig. 1). One thousand and eighteen nest boxes suitable for great tits are installed at this site, with the vast majority of great tits breeding in boxes. Individuals are trapped as nestlings and breeding adults at nest boxes, and are fitted with both a British Trust for Ornithology metal leg ring and a plastic leg ring containing a uniquely identifiable passive integrated transponder (PIT) tag (IB Technology). There is a further mist-netting effort over autumn and winter to tag individuals that immigrate into the population, and we estimate that over $90 \%$ of individuals had been PIT-tagged at the time of the study ${ }^{21}$. In this population, great tits form loose fission-fusion flocks of unrelated individuals in autumn and winter. Flocks congregate at patchy food sources and can be observed at bird feeders fitted with PIT-tag-detecting antennae $e^{21,31}$ The experiments were conducted in eight sub-population areas within Wytham Woods that had relatively little short-term between-area movement of individuals (Extended Data Fig. 1). The work was subject to review by the Department of Zoology ethical committee, University of Oxford, and was carried out under Natural England licences 20123075 and 20131205.

Puzzle-box design. The experimental apparatus consisted of an opaque plastic box with a perch positioned in front of a door that could be slid to either side with the bill, to gain access to a feeder concealed behind. Video observations suggested that all great tits used their bill to move the door. The left side of the door was coloured blue, and the right side was coloured red, with a raised front section on the door to allow an easier grip. The concealed feeder contained approximately 500 live mealworms and was refilled up to twice daily. Mealworms are a highly preferred food for great tits (Extended Data Fig. 5), and as live mealworms were used, solvers typically extracted one worm and then carried it away from the puzzle box to kill and eat it (as confirmed by video observations; Supplementary Videos 1 and 2). Each puzzle box was surrounded by a $1 \times 1 \mathrm{~m}^{2}$ cage with a $5 \times 5 \mathrm{~cm}^{2}$ mesh that allowed unlimited access by small birds but prevented access by large non-target species such as corvids or squirrels. A freely accessible bird feeder filled with peanut granules was also provided in the cage, at approximately $1 \mathrm{~m}$ from the puzzle box. Peanut granules are a much less preferred food source (Extended Data Fig. 5). Each peanut feeder had two access points fitted with RFID antennae and data-logging hardware. This feeder was used to attract the original demonstrator to the location and to record the identity of individuals that did not contact the puzzle box.

All puzzle boxes contained a printed circuit board and motor and were powered by a $12-\mathrm{V}$ sealed battery. The perch also functioned as an RFID antenna that registered the visit duration (the time to nearest second) and the identity of the visiting individual. A 'solve' was recorded if the door was opened during an individual visit to the device, with the side direction also noted. If a solve occurred without an accompanying identified individual, this was recorded as an 'unidentified solve' One second after the solving bird departed, the door reset itself back to the middle. If more individuals visited before this happened, then a 'scrounge' was recorded, as these individuals were assumed to have taken food from the open door (as confirmed by video observations). The door reset immediately after two individuals were registered scrounging, preventing more than two possible scrounging events per solve (Supplementary Video 2).

Experimental procedure. Two males were captured from each sub-population (11 adults and 5 juveniles) to act as demonstrators. They were captured either by removal from roosting boxes on Sunday night or by mist-netting at a sunflower-seed feeder on Monday morning. They were transferred to individual cages in indoor captive facilities, and over 4 days, each pair of birds was subjected to one of three training regimens using step-wise shaping: (i) given no training and left in the cage with ad libitum food (control); (ii) trained to solve the novel puzzle box by pushing the blue side of the door to the right (option B); or (iii) trained to solve the novel puzzle box by pushing the red side of the door to the left (option A). With the exception of 'control' areas, which were clustered in the south of the woodland to avoid cross-contamination, sub-populations were randomly assigned to a training regimen, with both demonstrators from a single sub-population trained on the same technique. During training, the demonstrators were initially exposed to an open puzzle box baited with mealworms, which was then gradually closed over the course of 4 days until the subjects were reliably re-opening it. The other side of the door was fixed during training. On Friday morning, the birds were released back at the site of capture in each respective sub-population. Puzzle boxes for which both options were available and were equally rewarding were installed at three sites $250 \mathrm{~m}$ apart on the following Sunday night (Extended Data Fig. 1). These puzzle boxes were run over a 4-week period at each site, continuously operating from Monday to Friday and then removed on Saturday and Sunday, for a total of 20 days of data collection.

Four replicates were conducted in the first year of data collection (December 2012 to February 2013; C1, C2, T1 and T3). At the sites for three of these replicates (C1, $\mathrm{T} 1$ and $\mathrm{T} 3$ ), the puzzle boxes were simultaneously re-installed at the same locations for 5 days of further data collection in December 2013. No additional demonstrators were trained, and no individual had had contact with the puzzle box in the 9 months between the two data collection periods. This second exposure sought to test the long-term stability of social learning at the sub-population level. This study was run before the second year of data collection for the cultural diffusion experiment, to exclude the possibility that dispersing individuals in new replicates could be re-introducing the novel behaviour. An additional four replicates (C3, T2, T4 and T5) were then conducted from December 2013 to February 2014 in new subpopulations, using the same initial protocol.

Data analysis. The local population size for each replicate was defined as comprising all individuals in a replicate that had been recorded at least once at one of the following: the puzzle box, the nearby peanut feeder or the nearest network-logging feeders (operated Saturday and Sunday, see below), during the experimental period (that is, from the weekend following the release of the demonstrators to the weekend after day 20 of operation of the puzzle boxes). When three replicates were compared with the 'persistence' trial in the following year, the local population was defined as all individuals observed at the puzzle box or as all individuals nearby the peanut feeder so that the areas were comparable.

To analyse the results of the initial experiment, we first compared control replicates and treatment replicates, by using Welch's two-sample $t$-tests and by fitting linear and sigmoidal models to the data, with the best model ascertained by the difference in the AIC values ${ }^{32}$. If individuals were using social information when learning about the puzzle box, then we expected that there would be a difference between the areas seeded with a trained demonstrator (treatment) and those with out (control). The replicates were thus compared in terms of latency to first solve (the number of seconds from the beginning of the experimental period, excluding demonstrator) and the total number of solutions. Second, we compared the total number of solutions in the two different experimental treatments. In this case, if a more complex form of social learning than local enhancement to the feeding site was occurring, then we expected a consistent bias towards the seeded variant in the different treatments ${ }^{14}$

To analyse the change in individual and population preferences for option A or $B$ over time, we used a generalized estimating equation (GEE) model ${ }^{2}$ where the dependent variable was the proportion of solutions using the seeded technique on each day of data collection and the explanatory variables were individuals and replicates, weighted by the overall number of solutions per day. The seeded technique (A or B) was initially also included as an explanatory variable but was not sig nificant (coefficient \pm s.e.m. $=0.13 \pm 0.22, P=0.55$ ). Three individual variables were included in a GEE model: sex, age and natal origin. Sex was determined at capture using plumage coloration; age was determined from breeding records or plumage coloration; and individuals were classed as 'immigrants' if they had dispersed into the study site and 'locally born' if they had been ringed as a nestling in the study site ${ }^{27}$. Only age was significant (coefficient \pm s.e.m. $=-0.92 \pm 0.20$ $P<0.001$ ) and was included in the final model (sex, coefficient \pm s.e.m. $=0.38 \pm 0.22$ $P=0.08$; natal origin, coefficient \pm s.e.m. $=-0.38 \pm 0.22, P=0.08$ ).

If population-level conformity was partly the result of a conformist transmission bias, then at first acquisition we would expect a sigmoidal relationship between the population-level frequency of the option and the probability of adoption, with adoption of the majority option disproportionately more likely than its absolute frequency. By contrast, copying the last individual observed, or random copying, should yield a linear relationship ${ }^{25,26}$, with the probability of adopting option A or $\mathrm{B}$ being roughly equal to its proportion in the overall population. To investigate this, we isolated the first observed solutions by all individuals in all experimenta replicates and compared the option choice to the proportion of all previous option A solves observed in the individual's group at that site. The group length was set at $245 \mathrm{~s}$, which was the average group length observed using Gaussian mixture models on temporal patterns of flocking (see below) at network-logging sunflowerseed feeders. Both linear and sigmoidal models were then fitted to the data, with the best model ascertained by the difference in AIC values ${ }^{32}$.

We further examined the subset of individuals that moved between sub-populations $(n=41)$. This subset included all individuals recorded in more than one experimental replicate, whether within the season $(n=17)$ or between seasons $(n=24)$. No individual was observed in more than two replicates, and this analysis did not include individuals in the 'persistence' trial. A preference for option A or B at each location was defined as more than $75 \%$ of all solves for either option A or B in that replicate. Finally, to analyse the change in within-individual bias towards option $\mathrm{A}$ or B between the initial experiment and the second-year persistence trials, we used a general linear model where the dependent variable was the number of solves using the seeded variant over the total number of solves for each individual observed in both years. The explanatory variables were treatment type and year, with individual identity as a random effect.

Network data collection and analysis. Sunflower-seed bird-feeding stations had been deployed at 65 locations around Wytham Woods on an approximately $250 \times 250 \mathrm{~m}^{2}$ grid, as part of long-term research into social network structure in tits ${ }^{21,22}$. Each 
station has two access points, each fitted with RFID antennae and data-logging hardware. The feeding stations automatically opened from dawn to dusk on Saturday and Sunday, scanning for PIT tags every $1 / 16 \mathrm{~s}$. This study used the data from the eight nearest locations to each set of puzzle boxes, for ten dates within and surrounding the cultural diffusion experiment (the standard logging protocol runs from September to February in Wytham Woods ${ }^{21}$ ).

Great tits were detected visiting feeding stations and were individually identified by their PIT tags. We then applied a Gaussian mixture model to the spatio-temporal data stream to detect distinct clusters of visits. This method locates high-density periods of feeding activity, thereby isolating flocks of feeding birds without imposing artificial assumptions about group boundaries ${ }^{22,33}$. A gambit of the group approach ${ }^{34}$ was used with a simple-ratio index to calculate social associations, where individual association strengths (network edges) were scaled between 0 (never observed foraging together in the same group) to 1 (always observed in the same group and never observed apart). While a single co-occurrence may not be meaningful, our automated data collection method resulted in thousands of repeated group sampling events, allowing social ties between individuals to be built up from multiple observations of co-occurrences over time and across spatial locations. The networks contained 123 (T1), 137 (T2), 154 (T3), 95 (T4) and 110 (T5) nodes; the average edge strengths were 0.09 (T1), 0.05 (T2), 0.08 (T3), 0.07 (T4) and 0.07 (T5). To test whether the networks contained significantly preferred and avoided relationships, we ran permutation tests on the grouping data, controlling for group size and the number of observations, restricting swaps within days and sites ${ }^{35,36}$. We tested whether the observed patterns of associations were non-random by comparing the coefficient of variance in the observed network with the coefficient of variance in the randomized networks ${ }^{35}$. The social networks for all replicates were significantly nonrandom, even at local scales (T1, $P<0.0001 ; \mathrm{T} 2, P=0.0005$; T3, $P<0.0001$; T4, $P=0.0002$; and T5, $P=0.0002$ ).

Finally, we used network-based approaches to ask whether the behaviour was socially transmitted through foraging associations. Network-based diffusion analysis
(NBDA) is an approach that tests for social learning by assuming that if social transmission is occurring, then the spread of trait acquisition should follow the patterns of relationships between individuals, with the transmission rate being linearly proportional to the association strength ${ }^{23,37,38}$. We used NBDA R code v.1.2 (ref. 38), with the time of each individual's first solution (the number of seconds since the beginning of the experiment) entered into the continuous time of acquisition analysis function. Individuals that solved the puzzle box but that did not appear in the social network (that is, that had not been recorded in the standardized weekend logging) were excluded from the analysis. The effects of three individual-level variables were also incorporated into the analysis: sex, age and natal origin. All combinations of NBDA provided in the NBDA R code v1.2 were run, with the social transmission rate allowed to vary for each replicate. An AIC model averaging approach was used to find the best-supported model ${ }^{38}$.

31. Aplin, L. M., Farine, D. R., Morand-Ferron, J. \& Sheldon, B. C. Social networks predict patch discovery in a wild population of songbirds. Proc. R. Soc. B 279, 4199-4205 (2012).

32. Burnham, K. P. \& Anderson, D. R. Model Selection and Multimodel Inference: A Practical Information-Theoretic Approach 2nd edn (Springer, 2002).

33. Farine, D. R. Animal social network inference and permutations for ecologist in $\mathrm{R}$ using asnipe. Methods Ecol. Evol. 4, 1187-1194 (2013).

34. Franks, D. W., Ruxton, G. D. \& James, R. Sampling animal association networks with the gambit of the group. Behav. Ecol. Sociobiol. 64, 493-503 (2010).

35. Whitehead, H. Analyzing Animal Societies: Ouantitative Methods for Vertebrate Social Analysis (Univ. Chicago Press, 2008).

36. Bejder, L., Fletcher, D. \& Brager, S. A method for testing association patterns of social animals. Anim. Behav. 56, 719-725 (1998).

37. Franz, M. \& Nunn, C. L. Network-based diffusion analysis: a new method for detecting social learning. Proc. R. Soc. B 276, 1829-1836 (2009).

38. Hoppitt, W., Boogert, N. J. \& Laland, K. N. Detecting social transmission in networks. J. Theor. Biol. 263, 544-555 (2010). 


\section{RESEARCH LETTER}

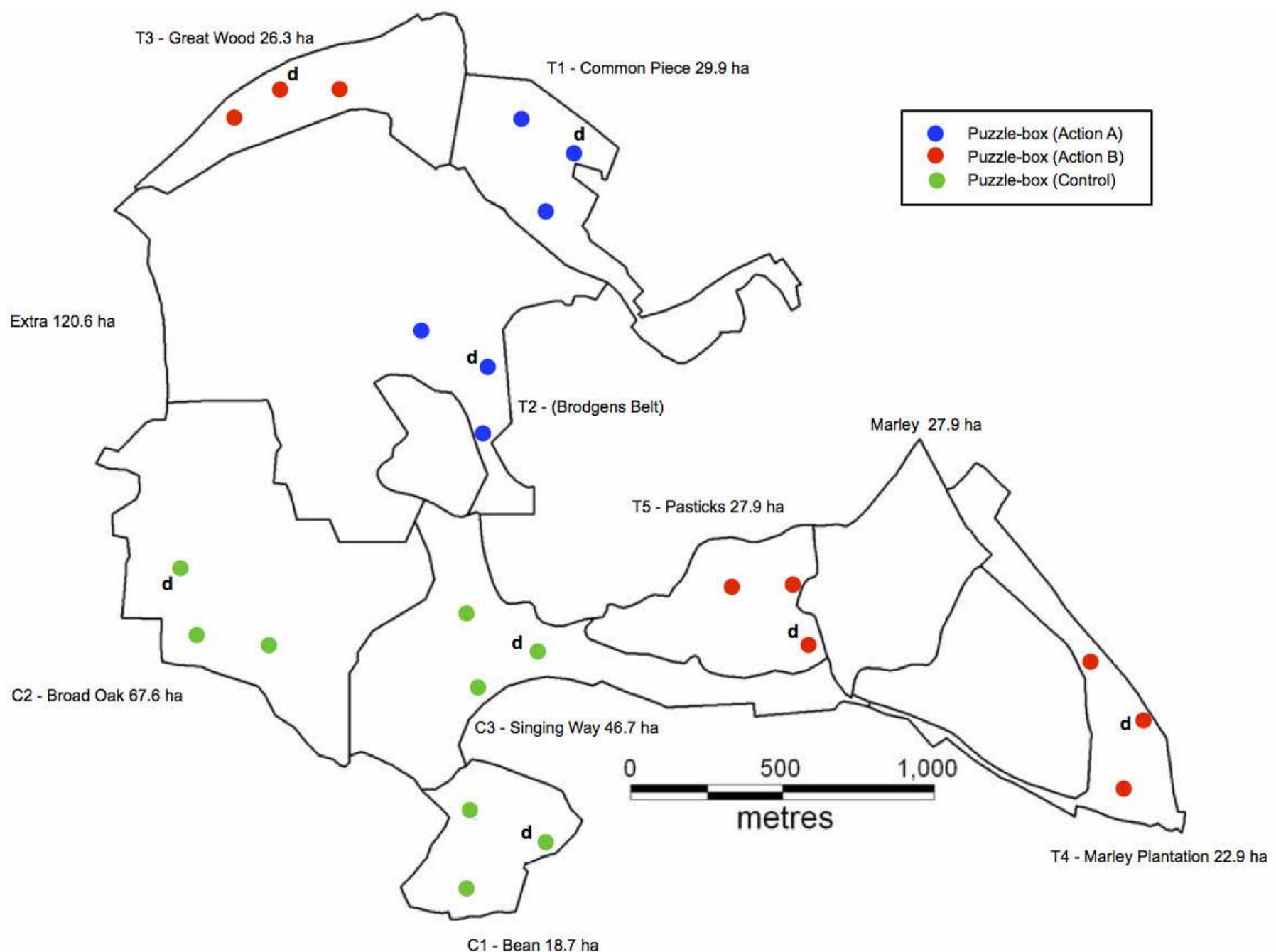

Extended Data Figure $1 \mid$ Wytham Woods, UK, $\left(51^{\circ} 46^{\prime} \mathrm{N}, 01^{\circ} 20^{\prime} \mathrm{W}\right)$, showing the location of replicates and puzzle boxes. The total area of

puzzle-box locations for the two option A replicates, T1 and T2: Common Piece and Brogden's Belt, respectively. The red points indicate the puzzle-box Wytham Woods is $385 \mathrm{ha}$; the location and size of the separate woodland areas within the woods are labelled on the map. The green points indicate the puzzle-box locations for the three control replicates, C1, C2 and C3: Broad Oak, locations for the three option B replicates, T3, T4 and T5: Great Wood, Marley Plantation and Pasticks, respectively. (d) indicates the locations where trained demonstrators were caught from and released to. 
a

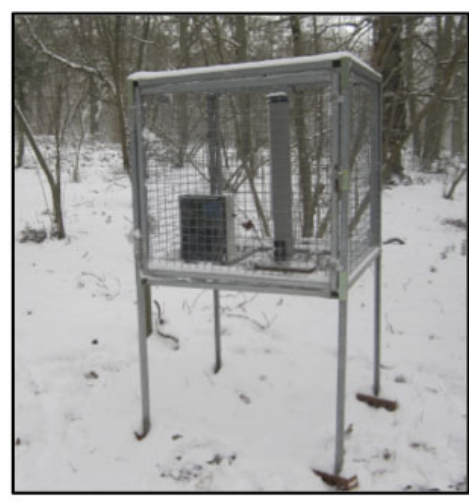

C

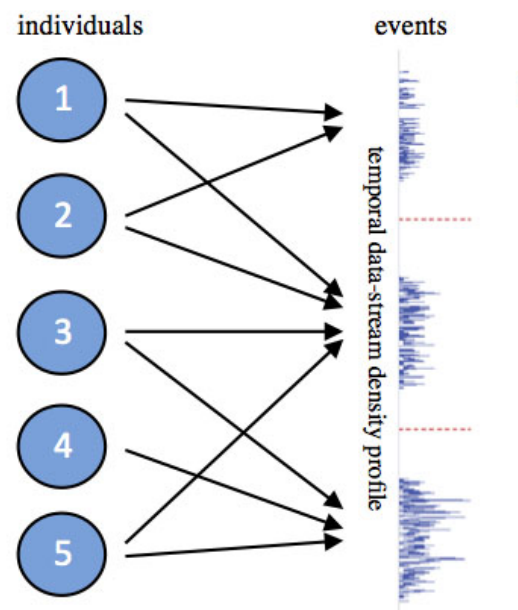

Extended Data Figure $2 \mid$ Social network data collection. a, Schematic of a feeding station (shut), with sunflower-seed feeder, RFID antennae and data-logging hardware. The cage is to restrict access to small passerines only. b, Map of the study area showing the placement of 65 feeding stations. The stations are approximately $250 \mathrm{~m}$ apart and open simultaneously from dawn to

d b
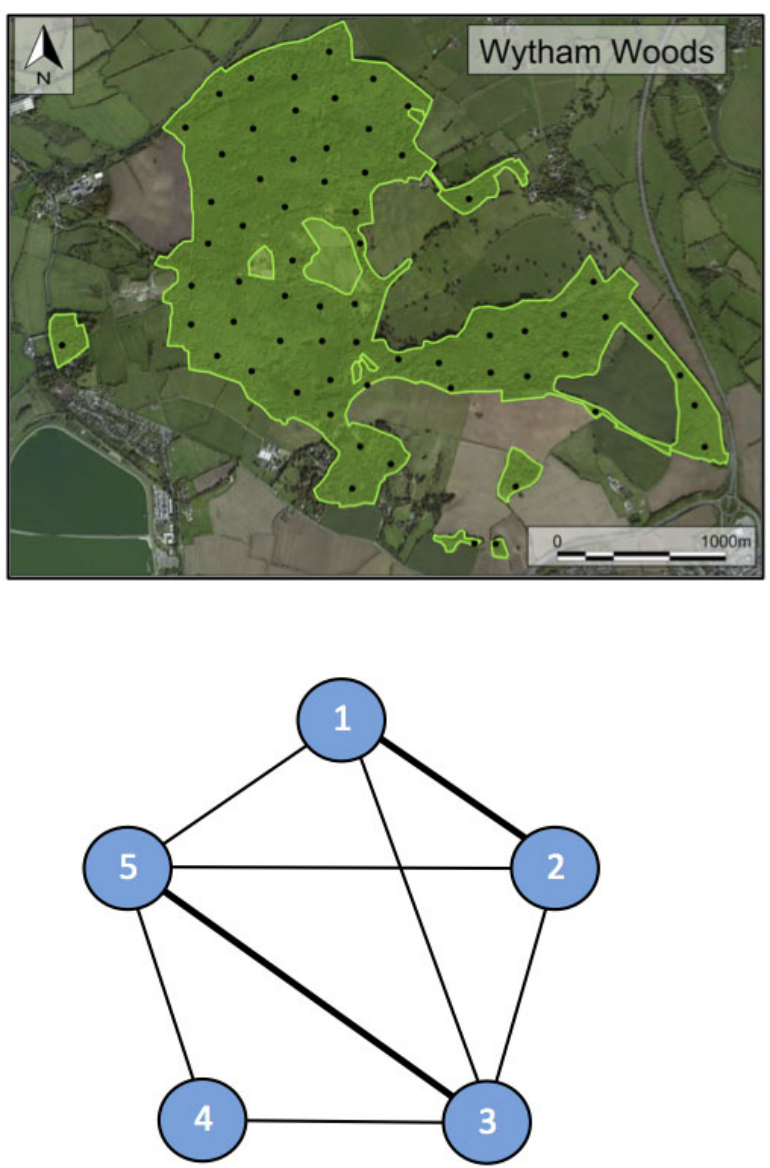

dusk on Saturday and Sunday over winter. c, Grouping events were inferred from the temporal data stream gained from the feeding stations, with individuals assigned to grouping events in a bipartite network. d, Repeated co-occurrences were used to create social networks ${ }^{22}$. 


\section{RESEARCH LETTER}

a

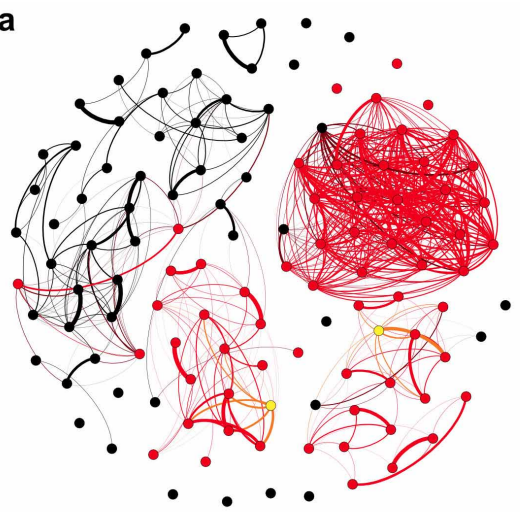

C

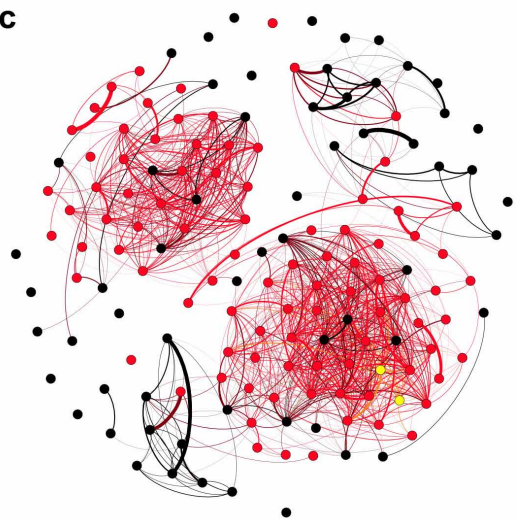

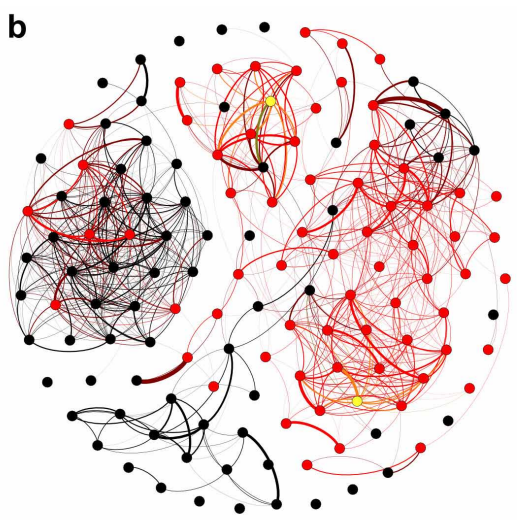

d

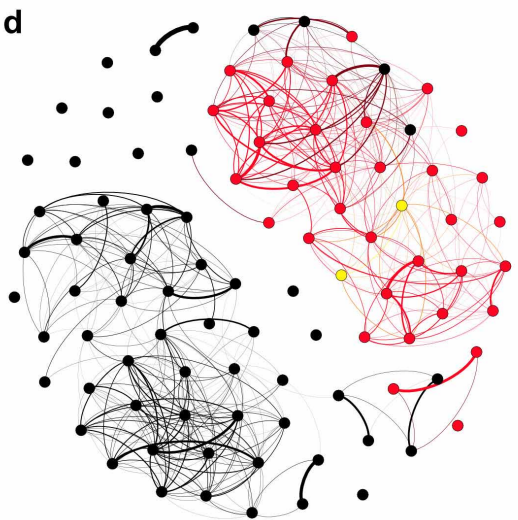

e

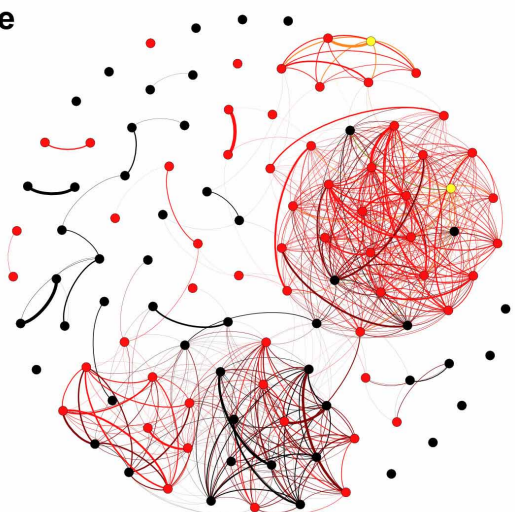

Extended Data Figure $3 \mid$ Social networks showing the diffusion of innovation. The red nodes represent individuals that acquired the novel behaviour after 20 days of exposure. The black nodes represent naive individuals. The yellow nodes represent trained demonstrators. The networks are heavily thresholded to show only the links above the average edge strength for each replicate (T1-T5, 0.09, 0.05, 0.08, 0.07 and 0.07, respectively). a, Network for the T1 replicate $(n=123)$. b, Network for the T2 replicate $(n=137)$. c, Network for the T3 replicate $(n=154)$. d, Network for the T4 replicate $(n=95)$. e, Network for the T5 replicate $(n=110)$. 
a

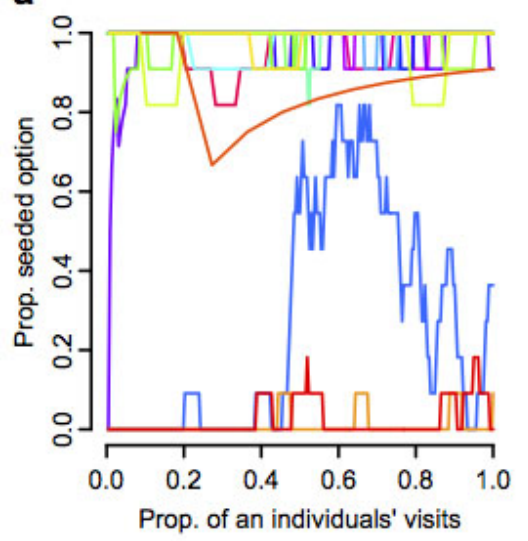

C

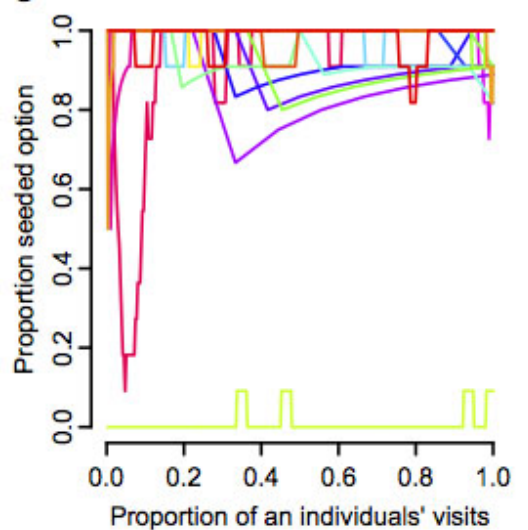

b

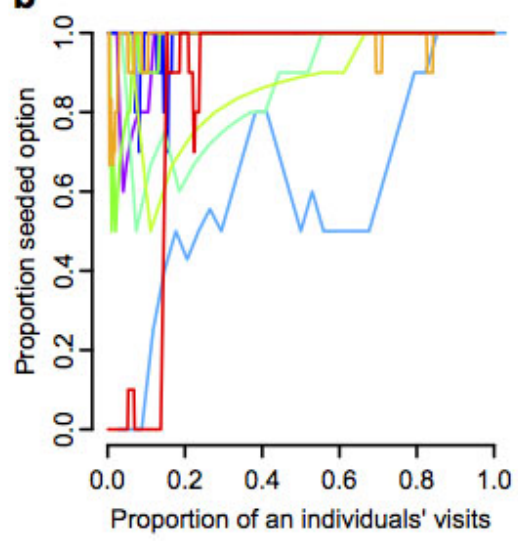

d

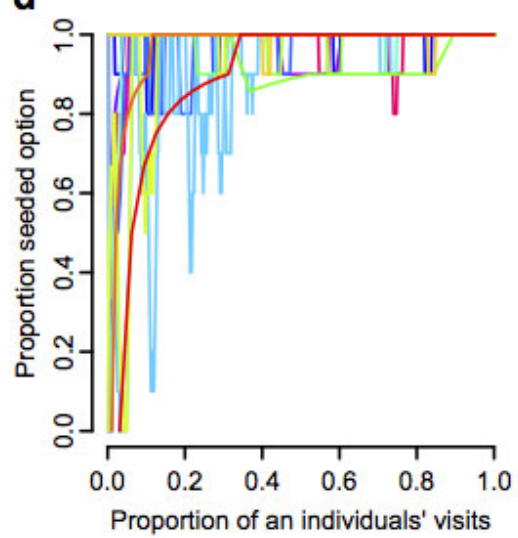

e

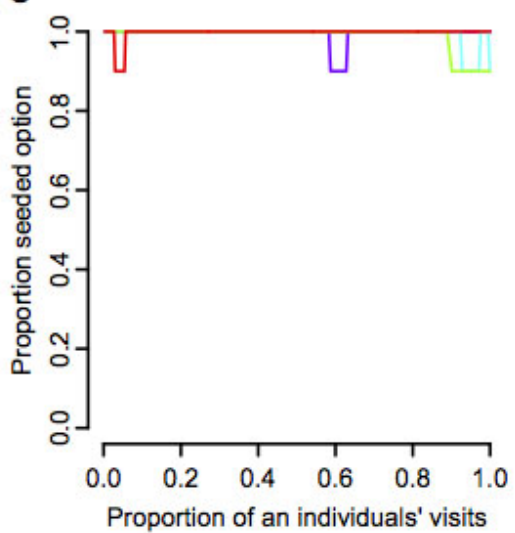

Extended Data Figure $4 \mid$ Individual trajectories (option A or B) for each replicate. Only individuals that performed both options are included, and individuals that moved between replicates are excluded. The lines are running proportions of the seeded option for each individual over its last ten visits. a, T1 (option A), $n=30$. b, T2 (option A), $n=10$. c, T3 (option B), $n=19$. d, T4 (option B), $n=15$. e, T5 (option B), $n=4$ 


\section{RESEARCH LETTER}

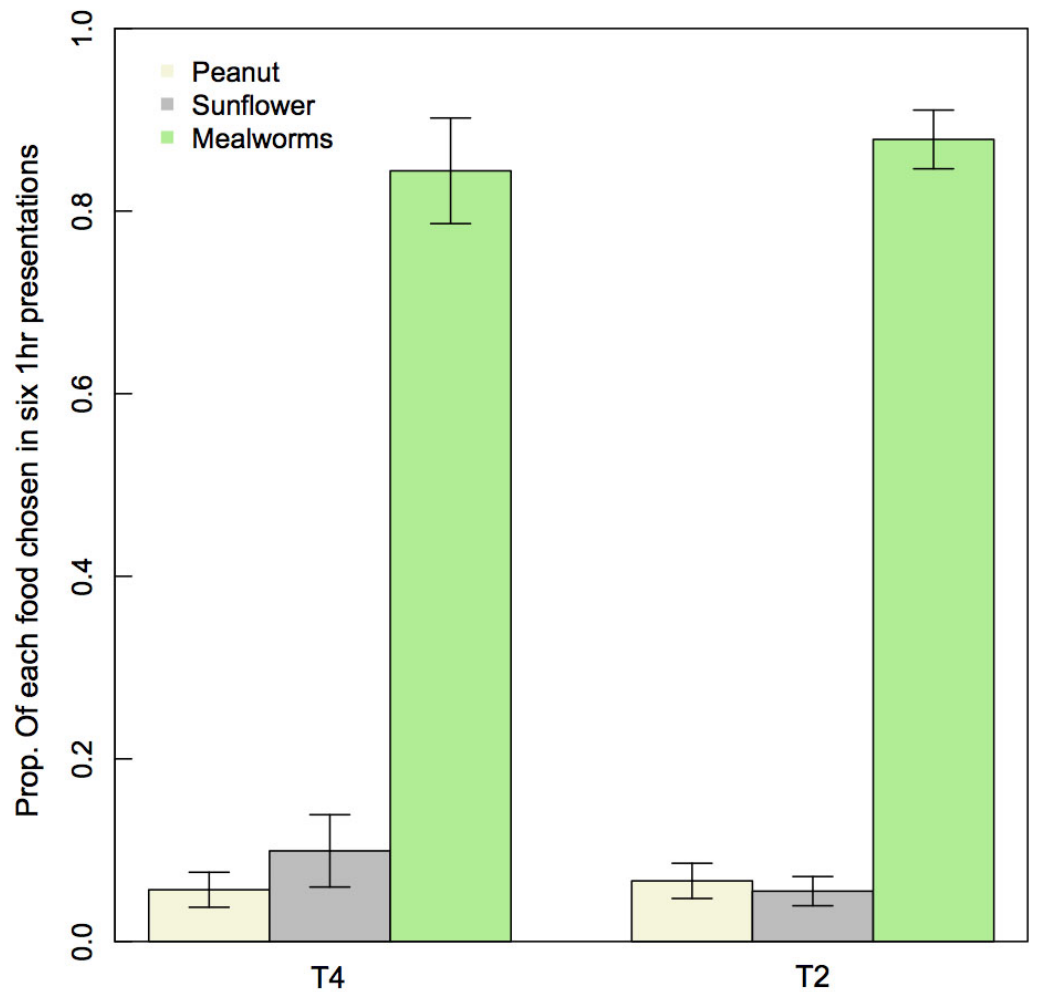

Extended Data Figure $5 \mid$ Food preference trials. The birds were presented with a freely available mix of 40 mealworms, 40 peanut granules and 40

in March 2014. Food choice was identified from video camera footage, and the trial was halted when all of one prey item was taken. Only great tits were sunflower seeds for $1 \mathrm{~h}$ on 2 days over 1 week at 6 sites ( 3 sites for T2 and 3 sites included, but the birds could not be individually identified. The birds clearly for T4). The trials were conducted 2 weeks after the end of the main experiment, preferred the live mealworms to peanut granules or sunflower seeds. 